\title{
Nocardiosis pulmonar como causa de reagudizaciones en un paciente EPOC
}

\author{
J. GUTIÉRREZ DUBOIS, J. L. ALONSO MARTÍNEZ, M. T. ÁLVAREZ FRÍAS, \\ M. L. ABÍNZANO GUILLÉN, L. MUNUERA GARCÍA, M. SOLANO REMÍREZ
}

Servicio de Medicina Interna. Hospital de Navarra. Pamplona

PULMONARY NOCARDIOSIS DUE TO REAGUDIZATIONS IN A COPD PATIENT

\section{RESUMEN}

La infección pulmonar por Nocardia sp. es una enfermedad poco frecuente que afecta fundamentalmente a pacientes inmunodeprimidos, aunque también puede hacerlo a pacientes inmunocompetentes. Su diagnóstico se basa en el aislamiento en esputo de Nocardia sp. siendo la clínica y la radiología inespecíficas. El tratamiento se realiza con trimetropin (TMP) sulfametoxazol (SMX), aunque ya se han encontrado casos de resistencia. La duración del tratamiento sigue siendo desconocida recomendándose durante 6 semanas-12 meses.

Presentamos el caso de un varón de 81 años con antecedentes de EPOC en tratamiento con corticosteroides de forma crónica que ingresa en nuestro servicio por episodios febriles recidivantes en los tres meses previos al ingreso junto con pérdida de peso e infiltrados densos en Rx de tórax de nueva aparición con cultivo de esputo positivo para Nocardia $s p$. Y buena evolución tras el inicio de tratamiento con TMP-SMX con desaparición de la fiebre y de los infiltrados.

PALABRAS CLAVE: Nocardia $s p$. Nocardiosis pulmonar. Neumonía bacteriana. EPOC.

\section{ABSTRACT}

Pulmonary infection due to Nocardia sp. is an infrequent disease that affects principally to immunodefficient patients although it can be also seen in patients with normal immunity. Diagnosis is based in isolation of micro-organism in respiratory samples while clinical presentation and radiology are non specific. Treatment is made with trimethropim-sulfametoxazole (TMP/SMX), though resistance has developed in some patients. The recommended length of treatment is 6 weeks to 12 months depending on the immunitaly status.

We present the case of a male patient of 81 years old affected with COPD and treated with glucocorticoids in a chronic basis, who was admitted because relapsing fever episodes during 3 months before, weight loss and new hard pulmonary infiltrates with Nocardia sp. cultured sputum, and evolution to clinical, radiological and microbiologic resolution with TMP/SMX treatment.

KEY WORDS: Nocardia sp. Pulmonary nocardiosis. Pulmonary bacterial infection. COPD.

Gutiérrez Dubois J, Alonso Martínez JL, Álvarez Frías MT, Abínzano Guillén ML, Munuera García L, Solano Remírez M. Nocardiosis pulmonar como causa de reagudizaciones en un paciente EPOC. An Med Interna (Madrid) 2006; 23: 537-539.

\section{INTRODUCCIÓN}

El aumento en la supervivencia de los pacientes inmunodeprimidos como son los pacientes con SIDA, oncológicos, transplantados y en tratamiento con corticoides, ha llevado a un incremento en las infecciones por gérmenes que raramente afectan a pacientes inmunocompetentes, como ocurre en el caso de la infección pulmonar por Nocardia sp. (1,2). Generalmente se adquiere a través del tracto respiratorio, siendo $N$. Asteroides el microorganismo aislado más frecuentemente (85\% de aislamientos) $(2,3)$.
$\mathrm{Su}$ diagnóstico se basa en el aislamiento en esputo de Nocardia $s p$. aumentando la rentabilidad el lavado bronquioloalveolar (2). La sintomatología y radiología son inespecíficas, confundiéndose el cuadro en muchas ocasiones con procesos malignos con infección por M. tuberculosis, o infecciones por hongos, lo que conlleva un retraso importante en el diagnóstico con el aumento subsecuente de la morbimortalidad, los días de ingreso hospitalario y los costes $(4,5,13)$.

El tratamiento se basa en la administración de trimetropinsulfametoxazol, aunque ya se han encontrado casos de resisten-

Trabajo aceptado: 24 de mayo de 2006

Correspondencia: Jorge Gutiérrez Dubois. C/ Padre Barace, 1, 6. ${ }^{\circ}$ A, esc. dcha. 31007 Pamplona (Navarra). e-mail: miriano2001@ yahoo.es 
cia $(3,6)$. Otros fármacos activos y con sinergismo frente a nocardia son el Imipenem, la amikacina, la minociclina y las cefalosporinas de 3 generación (4,7). La duración del tratamiento continua siendo incierta recomendándose durante 6 semanas12 meses dependiendo de la afectación y del estado inmune del paciente.

\section{CASO APORTADO}

Presentamos el caso de un varón de 81 años con AP de cardiopatía isquémica, arritimia cardiaca por FA crónica, isquemia arterial crónica de EEII, aneurisma de aorta abdominal, episodio previo de AIT y EPOC de 20 años de evolución en tratamiento corticoideo crónico. El paciente había dejado de fumar hacía 20 años y no refería otros hábitos tóxicos. Recibía tratamiento con digoxina, amlodipino AAS omeprazol, prednisona $10 \mathrm{mg}$ diarios y broncodilatadores nebulizados. Ingresó desde el Servicio de Urgencias por presentar desde hacía tres meses episodios febriles recidivantes con temperatura hasta 38,5 acompañados de disnea, sudoración intensa, astenia tos y expectoración purulenta sin hemoptisis y pérdida de aproximadamente $10 \mathrm{~kg}$ de peso. Además en este tiempo había estado ingresado en dos ocasiones en otro servicio, por estos mismos episodios diagnosticándose de infección respiratoria inespecífica y descompensación de EPOC. Ultimamente refería dolor abdominal en epigastrio irradiado hacia ambos flancos junto con despeños diarreicos de pequeña cantidad siendo las heces de características normales. El paciente comentaba también poliaquiuria y nicturia. A la exploración física el paciente presentaba un regular estado general, sin datos de deshidratación y con ligera palidez mucocutanea. La temperatura era de $38,7^{\circ}$; la TA de 110/60 mm de $\mathrm{Hg}$, el pulso era arrítmico a $90 \mathrm{lpm}$ y saturaba respirando aire ambiente a $89 \%$. La auscultación cardiaca era arrítmica con los tonos apagados y a la auscultación pulmonar existía una hipoventilación generalizada con roncus dispersos movibles. No se palpaban los pulsos pedios en ambas EEII y existían edemas con fovea bimaleolares. El resto de la exploración era normal. La gasometría arterial basal en el S de Urgencias presentaba una p02 de $53 \mathrm{~mm}$ de $\mathrm{Hg}, \mathrm{pCO}_{2}$ de $39,5 \mathrm{~mm}$ de $\mathrm{Hg}, \mathrm{pH}: 7,48$ y SO $\mathrm{S}_{2}$ de $90 \%$. En el hemograma la $\mathrm{Hb}$ era de 11,4 g/dl, Htco: $33 \%$, leucocitos: $11 \mathrm{x}$ $10^{9}$ (neutrófilos $89 \%$ ), Plq: 298 x 10\%. La VSG era de 89 y la PCR de $18,4 \mathrm{mg} / \mathrm{dL}$. La bioquímica la función renal y hepática así como las hormonas tiroideas fueron normales, los marcadores tumorales y los anticuerpos antinucleares fueron negativos. El único dato llamativo era una hipoalbuminemia de 2,5 g/dL En la RX de tórax del ingreso se observaban infiltrados densos en LID que no aparecían en Rx de ingresos anteriores (Fig. 1) por lo que se solicitó TAC torácico en el que se observaron varias formaciones nodulares en ambos campos pulmonares siendo la mayor de $5 \mathrm{cms}$ junto con derrame pleural izdo y una adenopatía paraaórtica izda de $3 \mathrm{~cm}$ (Fig. 2). El estudio microbiológico que incluyó hemocultivos, cultivo de esputo y baciloscopias fue en principio negativo. Ante la sospecha de neoplasia pulmonar se practicó PAAF y biopsia de nódulo pulmonar con control ecográfico que resultó negativa para células neoplásicas, informándose como proceso inflamatorio granulomatoso. Dado que la sintomatología persistía pese a tratamiento antibiótico con amoxicilinaclavulamico a altas dosis y ante la sospecha de fiebre de origen tumoral se comenzó la administración de AINE (naproxeno) con desaparición de la fiebre, y de acuerdo con la familia se decidió no realizar más estudios ni tratamiento por lo que se dio de alta al paciente con seguimiento en consulta transcurridos 15 días. En este periodo llegó el cultivo de esputo positivo para Nocardia sp, por lo que se comenzó tratamiento con cotrimoxazol $1 \mathrm{cp}$ cada 12 horas y control en consulta a los tres meses, en dicho control la Rx de tórax se había normalizado, los parámetros inflamatorios habían disminuido en control analítico y el paciente no refería fiebre ni clínica infecciosa.

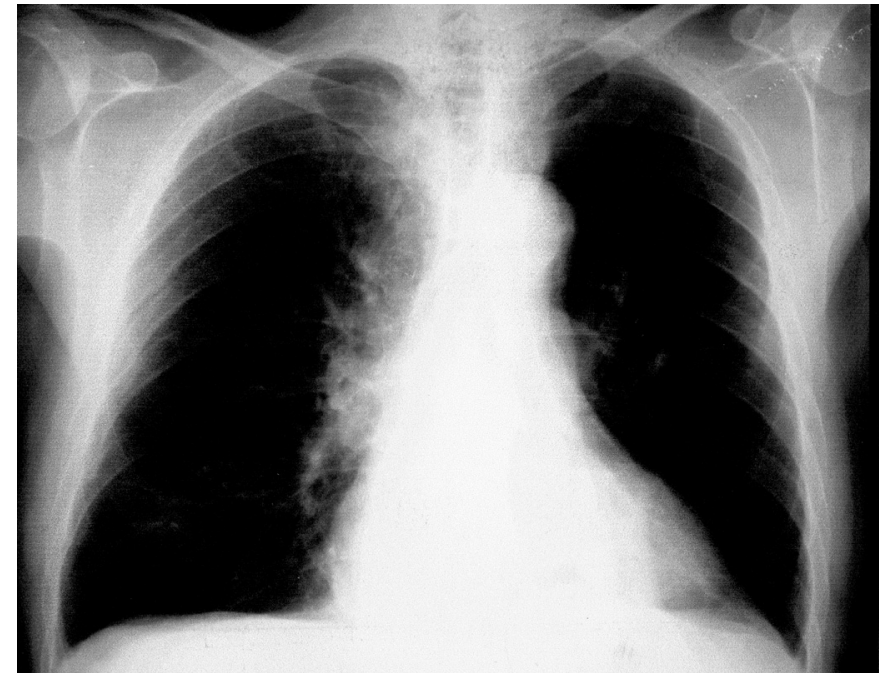

Fig. 1. Rx tórax.

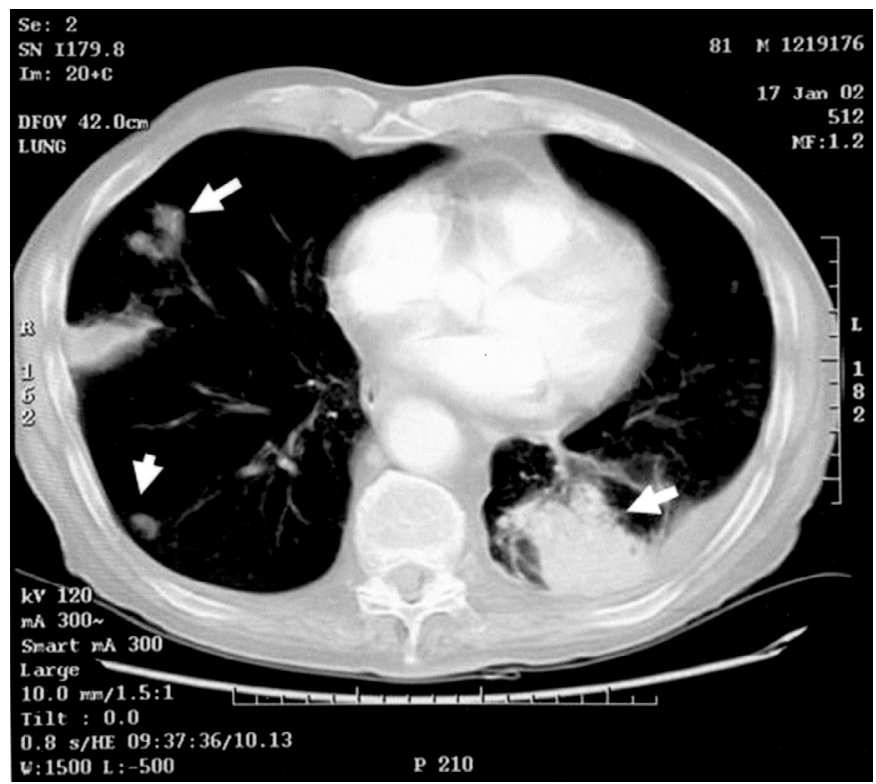

Fig. 2. TAC torácico.

\section{DISCUSIÓN}

Nocardia sp. es una bacteria aerobia gram + filamentosa y ramificada de distribución mundial y que se encuentra en la tierra y en la materia orgánica en descomposición. Comprende varias especies $N$. brasiliensis, $N$. transvalensis y $N$. pseudobrasielinsis, $N$. otitidiscavarium y $N$. asteroides, siendo esta última la que más frecuentemente afecta al hombre $(4,5)$. Generalmente se adquiere la infección a través del tracto respiratorio mediante inhalación de material contaminado, o mediante inoculación a través de la piel, no habiéndose demostrado contagio a través de contacto directo $(2,4,5)$.

La infección se da habitualmente en pacientes inmunocomprometidos sobre todo aquellos con afectación de la inmunidad celular como pacientes transplantados (tanto órganos sólidos como precursores hematopoyéticos), pacientes 
con enfermedades neoplásicas, con SIDA y pacientes con patologías que necesiten tratamiento corticoideo. Además los procesos que afectan a las defensas pulmonares del huésped (EPOC, Bronquiectasias, TBC, etc.) predisponen a la infección. En un 10-20\% de los casos los pacientes son inmunocompetentes $(1,2,9)$. Así en nuestro caso, el paciente había sido diagnosticado de EPOC hacía 20 años y recibía tratamiento crónico con corticoesteroides.

La nocardiosis pulmonar se caracteriza por ser generalmente un cuadro subagudo o crónico con tendencia hacia las remisiones y exacerbaciones. Los pacientes presentan habitualmente tos, fiebre, esputos purulentos y en algunas ocasiones hemoptoicos, astenia, anorexia y pérdida de peso (4). También se han descrito casos de síndrome de vena cava superior como forma de presentación (4). Radiológicamente las manifestaciones son variadas pudiendo aparecer infiltrados algodonosos, placas subpleurales, consolidaciones multilobares, masas solitarias, abscesos e infiltrados retículo nodulillares, siendo menos frecuente el derrame pleural (3, 10-12). Ni la clínica ni la radiología son lo suficientemente específicos para realizar el diagnóstico, y los pacientes en mucha ocasiones son diagnosticados inicialmente de TBC, neoplasia pulmonar o infecciones fúngicas $(4,5)$. En nuestro caso el paciente ya había estado ingresado previamente por dos episodios similares y en el ingreso presente se diagnosticó de proceso compatible con neoplasia pulmonar.

La infección pulmonar puede progresar hacia la aparición de un empiema (25\% de los casos) o hacia la diseminación sistémica con afectación de varios órganos, siendo el SNC el más frecuentemente afectado, hasta en un tercio de los casos $(3,12,14)$. Puede provocar además otras manifestaciones respiratorias como traqueitis, bronquitis, mediastinitis e infecciones cervicales (4).

El diagnóstico se basa en el aislamiento en el esputo de Nocardia sp. tal y como sucedió en nuestro paciente, aumentando la rentabilidad si se realiza un lavado bronquioalveolar aunque sólo está justificado cuando la sospecha es alta y el esputo no es diagnóstico (2). Son organismos de crecimiento lento pudiendo ser difícil su aislamiento e identificación en el laboratorio, como sucedió en nuestro caso que no fue hasta varios días después de que el paciente fue dado de alta que llegó el resultado del cultivo. Los hemocultivos suelen ser generalmente negativos. En la actualidad los tests serológicos no tienen utilidad debido a la escasa especificad por la reactividad cruzada con otras especies $(2,4,5)$.

Desde los años 40 el tratamiento de elección han sido las sulfonamidas. En la actualidad el antibiótico más usado es el Trimetropin (TMP)-Sulfametoxazol (SMX), ya que presenta actividad sinérgica frente a Nocardia sp, y atraviesa la barrera hemato-encefálica lo que le hace el fármaco de elección en casos de diseminación. No obstante se han documentado casos de aparición de resistencias de novo y fallo del tratamiento, pese a haberse demostrado sensibilidad in vitro, pudiendo deberse a que la concentración alcanzada del fármaco en los tejidos no es la adecuada $(3,6)$. En estos casos, otros fármacos que han demostrado actividad frente a Nocardia son Minociclina, Amikacina, Imipenem y cefalosporinas de tercera generación, habiéndose demostrado sinergismo entre ellos in vitro $(7,8)$. Pese al correcto tratamiento, no es desdeñable la mortalidad de la nocardiosis, sobre todo en su forma diseminada (44-85\%) dependiendo del estado inmune del huésped, habiéndose asociado mayor mortalidad a afectación del SNC $(80 \%)$ y tratamiento con corticoides (3).

La duración del tratamiento sigue siendo incierta, recomendándose entre 6 semanas en casos de pacientes inmunocompetentes, y en pacientes inmunodeprimidos o infección diseminada hasta 1 año. En nuestro paciente tras 3 meses de tratamiento las lesiones radiológicas habían desaparecido y clínicamente se encontraba afebril y sin clínica infecciosa (3-5).

La infección por Nocardia es una enfermedad poco frecuente que afecta generalmente a pacientes inmunodeprimidos y con patología pulmonar previa como es el caso que presentamos. En muchas ocasiones puede ser de difícil diagnóstico y confundida con otros procesos más frecuentes como cáncer de pulmón o TBC lo que provoca un retraso en su tratamiento, que lleva a un aumento en la estancia hospitalaria y en la morbi mortalidad de estos pacientes. Por ello, creemos que en pacientes como el del caso se debe tener presente la infección por Nocardia como posibilidad diagnóstica.

\section{Bibliografía}

1. Curry WA. Human nocardiosis: a clinical review with selected case reports. Arch Intern Med 1980; 140: 818-26.

2. Menéndez R, Cordero PJ, Santos M, Gobernando M, Marco V. Pulmonary infection with Nocardia species: a report of 10 cases and review. Eur Respir J 1997; 10: 1542-6.

3. Edith R, Nancy F. A case series and focused review of Nocardiosis. Medicine (Baltimore) 2004; 83: 300-13.

4. Lerner PI. Nocardiosis Clin Infect Dis 1996; 22: 891-903.

5. McNeil MM, Brown JM. The medically important aerobic actinomycetes: epidemiology and microbiology. Clin Microbiol Rev 1994; 7: 357 417.

6. Maderazo EG, Quintaliani R. Treatment of nocardial infections with trimethopin - sulfamethoxazole. Am J Med 1974; 57: 671-5.

7. Gombert ME, Aulicino TM. Synergism of imipenem and amikacina in combination with other antibiotics against Nocardia Asteroides. Antimicrob Agents Chemother 1983; 24: 810-1.

8. López FA, Johnson F, Novosad DM, Beaman BL, Holodny M. Succseful management of disseminated Nocardia transvalensis infection in a heart transplant recipient after development of sulfonamide resistance: case report and review. J Heart Lung Transplant 2003; 22: 492-7.

9. Daly, AS, McGeer A, Lipton JH, Systemic nocardiosis following allogenic bone marrow transplantation. Transplant Inf Dis 2003; 5: 16-20.

10. Dewey J, cances Jr. Bacterial pneumonia in inmunocompromised patients. J Thoracic Imaging 1998; 13: 261-70.

11. Yoon HK, Im JG, Ahn JM, Han MC. Pulmonary nocardiosis: CT findings. J Comput Assist Tomogr 1995; 19: 52-5.

12. Smilack JD. Pulmonary and disseminated nocardiosis. N Engl J Med 1999; 341: 885.

13. Kramer MR, Uttamchandani RB. The radiographic appearance of pulmonary nocardiosis asociated with AIDS. Chest 1990; 98: 382-5.

14. Kontoyiannis DP, Ruoff K, Hooper DC. Nocardia bacteraemia: report of 4 cases nd review of the literature. Medicine (Baltimore) 1998; 77: 255-67. 